\title{
Die Krise, die längst da war - Finanzkrise und soziale Ungleichheiten
}

\begin{abstract}
„Krise? Welche Krise?“ fragt Robert Misik in der taz ein Jahr nach Ausbruch der Finanzmarktkrise. Die Frage verweist auf einen doppelt-blinden Fleck in der öffentlichen Wahrnehmung der Finanzkrise, die sich nahezu weltweit zur Wirtschafts- und Sozialkrise entwickelt hat: Erstens gilt das Interesse einem dramatischen Ereignis und weniger seinen Ursachen und Folgewirkungen. Zweitens übersieht der Mittelschichten-Journalist - und ist damit nicht allein - die Menschen, die von den Folgen eines neuen Finanzmarktregimes schon lange betroffen sind, sich aber kaum zu Wort melden, vor allem nicht kollektiv. Sie sind, in unterschiedlicher Ausprägung und Interessenlage, sowohl ganz unten als auch in der Mitte der Gesellschaft zu finden. Was ist das Gemeinsame, was das Trennende in ihrer materiellen Lage und den dadurch geprägten Interessen?
\end{abstract}

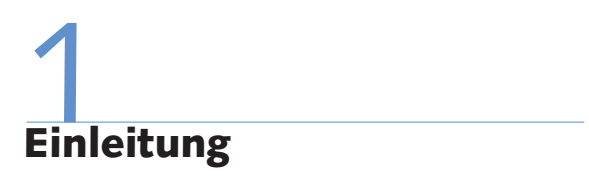

Das Ereignis liegt 22 Jahre zurück. Am Montag, den 19. Oktober 1987 erlebten die USA den bis dahin größten Börsenkrach seit 60 Jahren. In der New Yorker Wallstreet strömten Tausende zusammen, die Polizei bereitete sich auf Demonstrationen vor. Doch was geschah? „Verwirrt sahen die Polizisten, wie die Menschenmasse einfach dastand und nach oben starrte. Alle warteten darauf, dass die ersten verzweifelten Broker sich aus den Fenstern stürzen, keiner wollte das live event verpassen, den fröhlichen Augenblick, da die verfluchten Yuppies endlich auf den Asphalt prallen würden. Nun warteten sie und warteten sie, aber nichts geschah" (Paoli 2001, S. 75).

Dass nichts geschah, lag nicht nur daran, dass sich in den klimatisierten Büros der Wall Street kein Fenster mehr öffnen ließ. Warum die Menschenmenge das Winterpalais der Börsenwelt nicht erstürmte, sondern sich aufs Gaffen beschränkte, wirft Fragen auf, die sich auch heute wieder stellen. Damals mag sich in die banale Neugier der Zeitgenossen noch der Zorn der geprellten Aktienbürger und gar ein Hauch jener Volkswut gemischt haben, die der erschreckte Besitzbürger bisweilen noch den arbeitenden Klassen zutraut. Aber die Hierarchie der Betroffenen - von der Oberüber die Mittel- bis zur Unterschicht - verweist schon auf die zukünftige soziale Basis des weltweit expandierenden Finanz-Kapitalismus.

Was die Wucht der Ereignisse und den Umfang der materiellen Schäden betrifft, übertraf das Finanzbeben, das am 15. September 2008 nach der in New York besiegelten Pleite von Lehman Brothers einsetzte, den Crash von 1987 bei Weitem. Dennoch fanden bei dieser jüngsten, um den Globus ziehenden Finanzkrise kaum größere Massenaufläufe oder politische Demonstrationen statt, vom Fall der Northern Rock in England und von Island abgesehen. Diese, vielfach als Besonnenheit bezeichnete Ruhe wirft zwei Fragen auf, die ich im Folgenden behandeln will:

(1) Wie trifft die Finanzkrise verschiedene Klassen, Schichten und Menschengruppen? Unterscheidet sich nach der Krise das Spektrum der Betroffenheit von der Zeit davor? Oder treten die schon zuvor entwickelten Konturen einer öffentlich verdrängten Klassengesellschaft nur schärfer hervor?

(2) Wie erleben und verarbeiten die Menschen in den lohnabhängigen Unter- und Mittelschichten die für den FinanzmarktKapitalismus typischen Formen der gesellschaftlichen Spaltung? Welche klassen- und schichtenspezifischen Interessenkonstellationen zeichnen sich im Übergang von der Finanz- zur Sozialkrise ab?

Die Suche nach Antworten stützt sich vor allem auf empirische Befunde zur Entwicklung der sozialen Ungleichheit in Deutschland und auf einige Erwägungen zum möglichen Krisenbewusstsein in den unteren und mittleren Schichten der Gesellschaft. Die Deutungsversuche sind vorläufig und in Maßen spekulativ. Zumindest das haben sie mit ihrem Ausgangspunkt, der globalen Finanzkrise, gemeinsam.

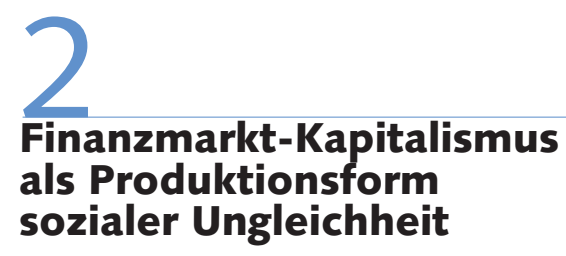

Wenngleich die Geld- und Kreditmärkte eine lange, schon immer mit Krisen einhergehende Geschichte kennen, so hat doch der in den letzten Jahrzehnten entstandene Regulationstyp des FinanzmarktKapitalismus eine besondere Dynamik entwickelt. Sein Einfluss auf die Struktur der sozialen Ungleichheiten wird hier am deutschen Beispiel skizziert; der an sich notwendige Blick auf die USA, das Epizentrum des letzten großen Finanzbebens, und auf die weltweiten sozialen Auswirkungen der Krise muss vernachlässigt werden. Natürlich sind die Veränderungen, die damit im Mittelpunkt stehen, nur vor dem Hintergrund der Zäsur von 1989 zu begreifen, wonach der Kapitalismus als herrschende Gesellschaftsformation übrig blieb. Zum ersten Mal in der Nachkriegszeit dringen seitdem Beschäftigungsformen und soziale Risiken, die für die Masse der Lohnarbeiter auch in krisenarmen Zeiten immer gegenwärtig waren, in den Erfahrungshorizont der abhängig beschäftigten Mittelklassen ein. Das wirkt übergreifend wie ein be-

Ulf Kadritzke, Prof. Dr., Hochschullehrer i. $R$. für Soziologie an der Hochschule für Wirtschaft und Recht (HWR), Berlin. Arbeitsschwerpunkte: Arbeits- und Betriebssoziologie, Mittelklassen und Management. e-mail: kadritzk@hwr-berlin.de 
drohliches Signal, weil bislang eine breite soziale Mitte die individuellen Aufstiegschancen durch Bildung und Leistung und damit die endgültige Überwindung der ,alten" Klassengesellschaft zu belegen schien. In welchem Zusammenhang stehen nun die Phänomene der verschärften Ungleichheit und einer verunsicherten Mitte mit der übergreifenden Entwicklung, die im Herbst 2008 eine weltweite Finanzmarktkrise mit schwerwiegenden Folgen für Wirtschaft und Gesellschaft auslöste?

Der Hintergrund lässt sich hier nur kurz andeuten. Das am Ende des Golden Age of Capitalism entwickelte „neoliberale Globalisierungsprojekt" (vgl. Herr in diesem Heft) hat, zunächst noch deutlich abgestuft nach den Wohlfahrtsregimen der einzelnen Staaten, einen Prozess in Gang gesetzt, der in allen entwickelten Ökonomien das Machtverhältnis zwischen Kapital und Arbeit neu justiert. Thatcherismus, Reaganomics und im letzten historischen Schub die politischen Umbrüche nach 1989 sichern der atlantischen Variante des Kapitalismus die entscheidenden Wettbewerbsvorteile gegenüber anderen, wohlfahrtsstaatlich gebändigten Modellen. In diesem Prozess verschieben sich auch die Machtgewichte zwischen den Wirtschaftssektoren. Vor allem die expandierenden Kapital- und Finanzmärkte werden zum stärksten Treiber der Globalisierungsprozesse. Die seitdem wachsende Kluft bei den Einkommen und Vermögen verweist auf die zwar nicht stärkste, aber doch maßgebliche Quelle des neoliberalen Projekts. Denn im Zuge wirtschaftlicher und institutioneller Veränderungen, der Erschließung neuer Kapitalressourcen und politischer Deregulierungsmaßnahmen (Deutschmann 2005; Kädtler 2005; Windolf 2005; Huffschmid 2009) fördert auch die breite Mobilisierung privater Geldvermögen die Dynamik der Finanzmärkte. Damit etabliert sich ein neues „Produktionsregime" (vgl. Dörre in diesem Heft), das vermittelt über globale Finanzströme die nationalen Beziehungen zwischen Lohnarbeit und Kapital verändert und den „Reichtum der Nationen" in deren Innern in einem Maße umverteilt, das im Golden Age nicht vorstellbar gewesen wäre.

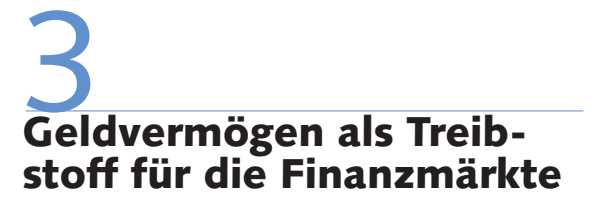

Aus dem nur grob skizzierten Wandel leitet sich der Gedanke ab, der im Folgenden weiter ausgesponnen wird: Wenn die Finanzmärkte in ihrer Ausrichtung auf die maximale Geldvermehrung das produktive Kapital und den Sozialstaat unter Druck setzen und die Politik dem nicht standhält, sondern noch den Weg bereitet, verschärfen sich die soziale Ungleichheit und die inhärenten Risiken des finanzgetriebenen Kapitalismus gleichermaßen. Die wachsenden Geldvermögen liefern den Schmierstoff für neue Strategien der Kapitalverwertung, die auf alle anderen Wirtschaftsbereiche und auf das Feld der bislang öffentlichen Dienste (Bahn, Post, Telekommunikation, Gesundheitswesen, Energie, Wasser) übergreifen. Das damit qualitativ veränderte Machtverhältnis zwischen Ökonomie und Politik bringt ein interner Investmentbericht nüchtern auf den Punkt: „War der Abbau von Beschäftigung bislang nur dann gesellschaftlich akzeptiert, wenn sich ein Unternehmen in wirtschaftlichen Schwierigkeiten befand, so kommunizieren Unternehmen nun offen Entlassungen und Gewinnsteigerung in einem Atemzug" (zitiert nach Thielemann 2009).

Nun sind diese Kapitalstrategien weit weniger von vermeintlichen Sachgesetzen bestimmt als ökonomische Analysen oftmals unterstellen. Das veränderte Kräfteverhältnis zwischen Kapital und Arbeit ist auch das Ergebnis politischer Regulation und der Konkurrenz zwischen unterschiedlichen Wohlfahrtsstaatregimen (so auch Herr in diesem Heft); und erst die phantasielose Selbstabdankung der Sozialstaatsparteien sichert der "Landnahme“ durch die Privatisierung des Sozialen den dauerhaften Erfolg. Kommt der Prozess freilich einmal in Gang, festigt er das neue Kapitalregime. Indem der Finanzsektor sich immer neue und größere Vermögensquellen erschließt, produziert er mit seinen radikalisierten Formen der Konkurrenz weltweit wachsende soziale Ungleichheiten (UN 2009).

Am stärksten treten diese Verschiebungen in den Heimatländern der großen Finanzmarktzentren zutage. In den USA hat sich seit 1980 nicht nur die Schere zwischen Arm und Reich (Krugman 2002), sondern auch zwischen den klassischen Industriezweigen und dem Finanzsektor geöffnet. Das Einkommen des Ingenieurs bleibt hinter dem des formal nicht höher qualifizierten Finanzspezialisten immer weiter zurück (Philippon/Reshef 2009). Das vertieft die Kluft zwischen den alten, professionellen Mittelklassen und einer spezialisierten Expertenschicht, die mit dem findigen Vertrieb neuer Anlagestrategien und Finanzprodukte zur Elite der Neureichen hinzustößt.

Dieser Zusammenhang gilt mit charakteristischer Verspätung auch für Deutschland, dessen Finanzdienstleistungssektor gesamtwirtschaftlich keine so große Rolle spielt wie in den USA und Großbritannien. Auch hier fördert das Vorbild der im Finanzsektor gezahlten erfolgsabhängigen Boni die Ungleichheit der Arbeitseinkommen in anderen Bereichen und trägt dazu bei, jenseits der Bankenwelt die allgemeine gesellschaftliche Kluft zu vertiefen.

\section{4 \\ Soziale Ungleichheit in Deutschland: schon vor der Krise verfestigt}

Die eingangs skizzierten Überlegungen zur Logik der Interessen und zur ökonomischen Macht, mit der die Akteure des Finanzmarkt-Kapitalismus die Ausweitung ihrer Anlagesphären betreiben, machen auch für Deutschland den Druck plausibel, der von der Ökonomie auf die Sphäre der Politik ausgeht. Die marktradikale Programmatik zielt auf die Verwandlung des öffentlichen Gutes Soziale Sicherheit: Wurde es bislang nach politisch gestalteten Gerechtigkeitsprinzipen und Verfahrensregeln, zumeist in nicht-marktlichen Formen der Kooperation und Organisation, zur Verfügung gestellt, so wird es jetzt zu einem auf dem Markt der privaten Dienstleistungen angebotenen Versicherungsprodukt. Die Öffnung gemeinschaftlich finanzierter Aufgaben für private Kapitalanlagen liegt einerseits in der Logik der wachsenden Konkurrenz auf globalen Finanzmärkten, sie ist andererseits auf nationalstaatlicher und regionaler Ebene (Beispiel EU) das Resultat einer politischen Willensbildung, die neben realen auch scheinbare Sachzwänge beschwört und mit dem moralischen Leitbild der Selbstverantwortung unterfüttert. Die strategische Weichenstellung ent- 


\begin{tabular}{|c|c|c|c|c|}
\hline \multicolumn{5}{|c|}{$\begin{array}{l}\text { Tabelle 1: Höhe des individuellen Vermögens in Euro nach Dezilen des } \\
\text { verfügbaren Haushaltseinkommens }{ }^{1}\end{array}$} \\
\hline \multirow{2}{*}{$\begin{array}{l}\text { Einkommensdezil } \\
\text { des Vorjahres }\end{array}$} & 2002 & 2007 & 2002 & 2007 \\
\hline & Median & Median & Mittelwert & Mittelwert \\
\hline 1. Dezil & 0 & 72 & 32.727 & 32.239 \\
\hline 2. Dezil & 2.916 & 2.365 & 41.846 & 37.760 \\
\hline 3. Dezil & 5.186 & 6.628 & 44.437 & 44.329 \\
\hline 4. Dezil & 8.933 & 9.942 & 42.607 & 48.589 \\
\hline 5. Dezil & 12.144 & 15.800 & 59.675 & 62.695 \\
\hline 6. Dezil & 20.000 & 19.580 & 67.890 & 60.268 \\
\hline 7. Dezil & 27.337 & 32.863 & 82.074 & 83.195 \\
\hline 8. Dezil & 31.463 & 36.057 & 87.563 & 97.896 \\
\hline 9. Dezil & 53.550 & 70.442 & 108.972 & 132.758 \\
\hline 10. Dezil & 125.559 & 140.658 & 257.019 & 317.072 \\
\hline Total & 17.962 & 21.624 & 80.055 & 88.034 \\
\hline \multicolumn{5}{|c|}{$\begin{array}{l}\text { 1) Äquivalenzgewichtete Haushaltsnettoeinkommen des Vorjahres unter Verwendung der modifizierten OECD-Äquivalenzskala } \\
\text { (exklusive Imputed Rent), Personen in Privathaushalten im Alter ab } 17 \text { Jahren. }\end{array}$} \\
\hline \multicolumn{5}{|c|}{$\begin{array}{l}\text { Quelle: Grabka/Frick 2009, S. } 64 \text { auf Basis des SOEP, individuelle Vermögensinformationen } \\
\text { nach multipler Imputation fehlender Werte, inklusive einem 0,1\% Top-Coding. }\end{array}$} \\
\hline
\end{tabular}

spricht gewiss dem ökonomischen Interesse mächtiger Akteure, zugleich ist sie jedoch von institutionellen, historisch gewachsenen Rahmenbedingungen geprägt und durch andere gesellschaftliche Kräfte beeinflussbar. Das heißt, politische Entscheidungen können innerhalb dieser Grenzen immer auch anders ausfallen. Das lehrt der Blick auf das nach wie vor bestehende Spektrum an staatlichen Wohlfahrtsmodellen.

Auch in Deutschland haben der verstärkte Druck der Finanzmärkte auf die Unternehmen und die Teilprivatisierung sozialstaatlicher Aufgabenbereiche den $\mathrm{Zu}$ schnitt und die Lebenschancen der sozialen Klassen und Schichten verändert. Das zeigt die Verteilung des gesellschaftlichen Reichtums. Das empirische Bild der Ungleichheit haben Sozialwissenschaftler in einem Sammelband über Deutschlands „gespaltene Gesellschaft" (Lessenich/Nullmeier 2006) aus unterschiedlichen Blickwinkeln erfasst und gedeutet. Die Einzelstudien weisen einhellig die klare Tendenz zur sozialen Polarisierung auf. Auch jüngste empirische Analysen verdeutlichen, in welchem Maß die Landnahme der Ökonomie und der Rückzug des Staates schon vor dem Ausbruch der globalen Finanzmarktkrise die Struktur der sozialen Ungleichheit geprägt und verändert haben. In Deutschland vermitteln vor allem die Längsschnittstudien des Sozio-oekonomischen Panels (SOEP) und der jährlich veröffentlichte Verteilungsbericht des WSI (für 2009: Schäfer in diesem Heft) ein empirisch tiefenscharfes Bild der gesellschaftlichen Entwicklung. Einige empirische Befunde seien im Folgenden herausgegriffen.

\subsection{UNGLEICHHEIT DER EINKOMMEN}

Die handfeste Grundlage der individuellen Lebensführung bildet das verfügbare Einkommen. Den deutlichsten Hinweis auf die Entwicklung der sozialen Ungleichheit liefert ein Blick auf die veränderte Zusammensetzung der Einkommensarten: Der Anteil der Einkommen aus abhängiger Beschäftigung, der Nettolöhne und -gehälter, ist schon in der alten Bundesrepublik von knapp 56 \% im Jahr 1970 auf 48,5 \% im Jahr 1990 und danach, in Deutschland insgesamt, weiter auf schließlich 38,4\% im ersten Halbjahr 2009 gesunken (vgl. Schäfer in diesem Heft). Auch die bereinigte Lohnquote, die den sich verändernden Anteil der abhängig Beschäftigten und Selbstständigen an allen Erwerbstätigen berücksichtigt, zeigt seit den 1980er Jahren eine fallende Tendenz. Von 1980 bis 1990 ist in der früheren Bundesrepublik der Anteil der Arbeitnehmereinkommen am realen Bruttoinlandsprodukt von knapp $70 \%$ auf unter $64 \%$ gefallen und zwischen 2000 und 2006 in Deutschland insgesamt, nach einem vereinigungsbedingten Zwischenhoch, von $67 \%$ auf $61 \%$. Seit dem Jahr 2000 sind hier die realen Arbeitnehmerentgelte entgegen dem europäischen Trend um $9 \%$ gesunken (Brenke 2009, S. 557f.; vgl. auch Ganßmann/Himmelreicher in diesem Heft). Hinter diesen Durchschnittswerten verbirgt sich eine noch weit dramatischere Verschiebung in der Verteilung des Reichtums.

Auf der Ebene der Einkommen belegt die jüngste, auf die Längsschnittdaten des SOEP gestützte Studie von Grabka/Frick
(2008) eine Tendenz zur „dauerhaften Polarisierung " und zur weiteren Verfestigung der sozialen Ungleichheitsstrukturen. Sie treten vor allem ganz oben und ganz unten zutage. Bei den „armutsgefährdeten“ Personen, die über $70 \%$ und weniger des Medianeinkommens verfügen und gut $25 \%$ der Bevölkerung ausmachen, stieg die Quote der Beharrung in der unteren Einkommensschicht von $54 \%$ im Jahr 2000 auf $66 \%$ im Jahr 2006 deutlich an. Noch stärker erhöhte sich diese Quote im obersten Segment der "Einkommensstarken“, die $150 \%$ und mehr des Medianeinkommens erzielen und gut $20 \%$ der Bevölkerung stellen. Von ihnen konnten im Jahre 2006 rund $69 \%$ ihre Einkommensposition halten, das sind nochmals fünf Prozentpunkte mehr als 2000. Die Wohlstandsgewinner haben damit ihre Einkommensposition weiter ausbauen können - und sie haben dies auch zulasten der mittleren Einkommensgruppen (mit $70 \%$ bis $150 \%$ des Medianeinkommens) getan. Der Anteil Letzterer ist seit 2000 von $63 \%$ auf $54 \%$ gesunken, zugleich hat sich im Innern der Mittelschicht der Prozess der Polarisierung verstärkt, denn gerade der Anteil der mittigsten Einkommen (90 \% bis unter $110 \%$ des Medians) ist in den letzten zehn Jahren von $19 \%$ auf $14 \%$ deutlich gesunken. In dieses Bild passt eine leichte Abwärtsbewegung der Mobilitätsrate. Während 2006 $11 \%$ der Personen aus der Einkommensmitte in die Schicht der Wohlhabenden aufgestiegen sind, sanken bei $14 \%$ die Einkommen unter die 70-\%-Marke des Medians in die Richtung der Armutsgefährdung ab. Dafür waren vor allem die Zunahme von Teilzeit- oder geringfügiger Beschäftigung und die Einführung des Arbeitslosengeldes II verantwortlich (Grabka/Frick 2008, S. 104).

\subsection{VERMÖGENSARME UND VERMÖGENSELITE}

In ihrer jüngsten Studie verdeutlichen Frick/Grabka (2009) die zusätzlich polarisierende Rolle der Vermögensverteilung. Schon der „Natur der Sache“ nach setzt die Umwandlung von Einkommen in Vermögen eine Mechanik der Ungleichheitsproduktion in Gang. Tabelle 1 zeigt, wie sich die Vermögen über die Bevölkerungsklassen verteilen.

Im Zahlenvergleich zwischen den Jahren 2002 und 2007 tritt die verschärfte Polarisierung der individuellen Nettovermö- 
gen zunächst in der extrem schiefen Verteilung der Durchschnittswerte über die zehn Einkommensklassen zutage, wobei in den beiden obersten Dezilen der 2007 nochmals deutlich steilere Anstieg des Mittelwerts herausragt. Im untersten Einkommenszehntel dagegen zeigt der Medianwert von $72 € \mathrm{im}$ Jahr 2007 an, dass nahezu $50 \%$ der Menschen in dieser Gruppe kein Vermögen gebildet haben oder sogar verschuldet sind. Nach den ergänzenden Befunden des SOEP verfügen knapp $27 \%$ aller Erwachsenen in Deutschland über keinerlei oder negatives Vermögen (Frick/ Grabka 2009, S. 65, Tabelle 4).

Im oberen Einkommenszehntel bedeutet der Medianwert (2007) von gut $140.000 €$, dass $50 \%$ der Einkommensreichsten unter und $50 \%$ über dieser Marke liegen, demgegenüber erreicht der Mittelwert dieser Gruppe die Höhe von $317.000 €$. Der binnen fünf Jahren (2002 bis 2007) erneut vergrößerte Abstand zwischen Median und Mittelwert verweist darauf, dass in Deutschland (wie in den USA) der Reichtum der Vermögenselite sich nochmals ganz oben in einer Spitzengruppe der Superreichen konzentriert: Das oberste Zehntel der Bevölkerung verfügt über mehr als $60 \%$ und die Gruppe der Reichsten $5 \%$ über $46 \%$ des gesamten deutschen Nettovermögens - aber allein das eine Prozent an der Spitze hat einen Anteil von $23 \%$ an sich gebracht. Diese Befunde der SOEP-Studien dürften die tatsächliche Konzentration des Reichtums an der Spitze noch unterschätzen (vgl. Frick/Grabka 2009, S. 58).

Bei den Vermögensverhältnissen in den Mittelschichten sind vielfältige Motive und Zwänge zu bedenken, die Selbstständige, Beamte, Angestellte oder Arbeiter mit unterschiedlichen Verdiensthöhen zum Vermögensaufbau bewegen. Anders als ganz unten kann erst auf der Basis mittlerer Einkommen Vermögen überhaupt angespart werden. In klarer Abstufung nach der Qualifikationshöhe liegt das Durchschnittsvermögen von Facharbeitern und einfachen Angestellten bei $44.000 €$, das von qualifizierten Angestellten und Beamten bis zum mittleren Dienst bei etwas über $65.000 €$, während Angestellte mit Führungsaufgaben $(116.000 €)$ und höhere Beamtenränge $(138.000 €)$ deutlich höher rangieren. In den mittleren Vermögensklassen sind zudem die Chancen der weiteren Geldvermehrung an die Stetigkeit des Beschäftigungsverhältnisses gebunden;

\section{Tabelle 2: Armut, Prekarität und Wohlstand in Deutschland}

\begin{tabular}{|c|c|c|c|}
\hline Zone von ... & $\begin{array}{l}\text { Personen } \\
\text { in \% }\end{array}$ & $\begin{array}{c}\text { Einkommen in \% des } \\
\text { Mittelwertes } \\
\text { im Jahre }\end{array}$ & $\begin{array}{l}\text { Anzahl der Notlagen }{ }^{1} \\
\text { chnitt }\end{array}$ \\
\hline ... gesichertem Wohlstand & 45,9 & 131,8 & 0,1 \\
\hline ... instabilem Wohlstand & 26,1 & 89,0 & 0,5 \\
\hline ... einseitiger Armut & 3,8 & 68,2 & 0,9 \\
\hline ... temporärer Armut & 5,7 & 68,6 & 1,1 \\
\hline ...Prekariat & 10,1 & 60,9 & 1,1 \\
\hline ... verfestigter Armut & 8,4 & 43,1 & 2,3 \\
\hline Total & 100,0 & 100,0 & 0,6 \\
\hline \multicolumn{4}{|c|}{$\begin{array}{l}\text { 1) Lesehilfe: Bestimmt werden vier Lebensbereiche (Wohnen, Teilhabe am Konsum, Rücklagenbildung und Arbeitslosigkeit), in } \\
\text { denen ein definierter Mindeststandard unterschritten wird. Bei Notlagen in allen vier Bereichen wäre der Höchstwert } 4 .\end{array}$} \\
\hline \multicolumn{4}{|c|}{$\begin{array}{l}\text { Quelle: Groh-Samberg 2007, S. 179, gestützt auf die Quellen: SOEP, Wellen Q bis U } \\
\text { (Längsschnittpopulation) N = 22 291; Berechnungen des DIW Berlin. }\end{array}$} \\
\hline
\end{tabular}

sie sinken in Phasen von Arbeitslosigkeit rasch, zumal die Sicherungsfunktion eines maßvollen Vermögens nicht beliebig oft zu nutzen ist.

\subsection{VERFESTIGTE ARMUT - VERSCHONTE MITTELSCHICHTEN?}

Ein nicht nur an Einkommen und Vermögen ausgerichtetes Gesamtbild der strukturierten Ungleichheit entwickelt Groh-Samberg $(2007,2009)$ in seinen theoretisch reflektierten und methodisch sorgfältigen Studien. Mit deren ernüchternden Befunden belegt er die Fortexistenz grundlegender, keineswegs entstrukturierter Klassenverhältnisse. Die in den letzten Jahren noch verfestigte Struktur der Ungleichheit in Deutschland tritt in der Verteilung der Menschen auf die Zonen von Armut, Prekarität und Wohlstand klar zutage (Tabelle 2). Die ergänzende Erhebung von Indikatoren für Notlagen in vier konstitutiven Lebensbereichen (Wohnen, Teilhabe am Konsum, Möglichkeit der Rücklagenbildung, Arbeitslosigkeit) liefert ein tiefenscharfes Bild über die Lebensbedingungen der „kleinen Leute“.

Der nüchterne Befund: Im Jahr 2004 umfasst die Zone extremer, „verfestigter Armut" 8,4 \% der Bevölkerung; die Zone der Prekarität (Armutsgefährdung bzw. teil- und zeitweise Armut) weitere 19,6\%. Damit leben schon lange vor dem Ausbruch der Finanzmarktkrise $28 \%$ der Menschen in Deutschland in verfestigter Armut oder prekären Lebensverhältnissen, in denen die Armut eine stets gegenwärtige Drohung ist. In diesen Zonen ist die Verbleibsquote zwischen 2000 und 2006 nochmals drastisch von 53,6 \% auf 66,2 \% gestiegen (Grabka/Frick 2008, S. 104), sodass ein wachsender Anteil von Menschen in der Falle der sozialen Exklusion auf Dauer gefangen ist. Das gilt vor allem dann, wenn zu den Hauptmerkmalen (unqualifizierter Beruf, Beschäftigung in Niedriglohnbranchen, Dauerarbeitslosigkeit) weitere depravierende Faktoren (z. B. der Status als Migrant oder alleinerziehende Mutter) hinzutreten. Erst über diesen beiden Bereichen liegen die Zone des „unstabilen Wohlstands", die $26 \%$ der Bevölkerung umfasst, und die des gesicherten Wohlstands (46\%).

\subsection{ZWISCHENFAZIT}

Die Befunde verdeutlichen, dass erst die am Ende des Golden Age einsetzende Polarisierung von Einkommen, die sich allenthalben in fallenden Lohnquoten ausdrückt, weltweit eine Anhäufung einseitig konzentrierter Vermögenswerte bewirkt, aus deren Quelle sich der Finanzmarkt-Kapitalismus nährt. Seine Renditevorgaben schmälern - sofern ihnen die Politik den Gefallen tut - die materiellen Ressourcen des Sozialstaats und damit die Möglichkeit, politisch ausgehandelte Prinzipien der Gerechtigkeit zu produzieren, zum Beispiel verfestigte Armut zu „enthärten“. In dieser Hinsicht hat es die Politik in Deutschland, die sich in wechselnden Regierungskoalitionen fast zwanghaft der Modernisierung und Reformen verschrieb, im internationalen Vergleich besonders weit gebracht, gibt es doch kein anderes „entwickeltes Land, in dem die Bildungschancen und die Chancen der sozialen Mobilität so stark von der sozialen Herkunft abhängen “ (Groh-Samberg 2007, S. 182). 


\section{5 \\ Mittelklassen als Opfer des Finanzmarkt-Kapitalis- mus?}

Im Gefolge der Finanzkrise hat sich die öffentliche Aufmerksamkeit der Mittelklasse zugewendet. Während die Armen nach der Krise lediglich arm bleiben, gilt die mit Lehman-Zertifikaten um Teile ihres Vermögens geprellte Informatikerin als eigentlich tragische Figur. Das legt die Frage nahe: Haben die lohnabhängigen Mittelklassen im Prozess der finanzgetriebenen Ungleichheitsentwicklung eher als Opfer, Aktivisten oder indirekte Profiteure zu gelten? Sind ihre Mitglieder aus objektiven Gründen verunsichert oder verspüren sie die Angst vor dem Abstieg, ohne ernsthaft betroffen zu sein? Legt man die vorgestellten empirischen Befunde zugrunde, so scheint es, also käme diese Mitte der Gesellschaft im eigenen Alltagsleben kaum oder allenfalls punktuell mit Armut in Berührung. Aber bei der Interpretation ist Vorsicht geboten. Nur auf Einkommen und Vermögen konzentrierte Analysen können in den sozialen Mittellagen auch dann undeutlich bleiben, wenn die 10-\%-Gruppe der selbstständigen Mittelschichten aus der Betrachtung ausgenommen wird. Tatsächlich hat die arbeitssoziologische Forschung wichtige Veränderungen aufgespürt, die für moderne Mittelklassenberufe die Rede von prekärem Wohlstand und sozialer Verwundbarkeit (Bude 2008; Vogel 2009, S. 170ff.) rechtfertigen, weil der Wandel der traditionell über Bildung und Beruf garantierten Lebensperspektiven offensichtlich ist. Auch er steht mit den finanzgetriebenen und staatlich umgesetzten Reformen am Arbeitsmarkt und im Beschäftigungssystem in enger Verbindung.

So hat sich im Zuge der Entstandardisierung allein von 2000 bis 2006 der Anteil der abhängigen Vollzeitbeschäftigung von $64 \%$ auf $55 \%$ verringert (Grabka/Frick 2008, S. 104). Damit dringen, nicht auf breiter Front, aber auch nicht mehr rudimentär, bislang ungewohnte Ungleichheitserfahrungen bis in die Mitte der Gesellschaft vor. In privaten wie auch öffentlichen Betrieben haben Probeanstellungen, verlängerte Praktika, Scheinselbstständigkeit und sogar geringfügige Beschäftigung Einzug gehalten. Solche Zumutungen prägen oft schon den Alltag junger, gut ausge- bildeter Menschen, die sie als Durchgangsstadium zur gesicherten Vollzeitstelle akzeptieren müssen. Zur gefühlten Verwundbarkeit trägt in den abhängig beschäftigten Mittelschichten ferner die Entdeckung bei, dass die Risiken der Arbeitslosigkeit zwar deutlich geringer sind als in den Zonen der Prekarität und der Armut, der Ernstfall jedoch auch für sie die Gefahr eines zügigen sozialen Abstiegs einschließt. Das Gemisch aus Erfahrungen, Wissen und Ängsten hat dazu geführt, dass in den subjektiven Dimensionen der SOEP-Messungen der Anteil derjenigen aus den (nach dem Einkommen definierten) Mittelschichten, der sich "große Sorgen" macht, im Jahr 2005 mit $26 \%$ einen neuen Höhepunkt erreicht hat.

Bezieht man die Mittelklassen in das Gesamtbild der Gesellschaft ein, bleibt freilich die Erkenntnis: Die Spaltung der Gesellschaft hat sich in Deutschland vor allem deshalb verfestigt, weil die maßgeblich die Klassenstruktur prägenden Merkmale (Einkommen und Vermögen, Bildungsgrad, Geschlecht, ethnische Zugehörigkeit) sich in der Wirkung wechselseitig verstärken. Selten nur können, und dann vor allem in der qualifizierten Mitte, einzelne Stärken wie eine gute Bildung die anderen Faktoren kompensieren. Entgegen dem herrschenden Leitbild der sozialen Mobilität, das insbesondere ein die individuelle Chancengleichheit förderndes Bildungssystem zu verwirklichen versprach, festigen und verstetigen sich sowohl die exklusive Position der Elite wie auch Armut und Prekarität in den unteren Rängen der Gesellschaft. An beiden Polen der Klassengesellschaft greift wieder der vormoderne Mechanismus der „sozialen Vererbung“, während in den gesellschaftlichen Mittellagen der finanzgetriebene Wandel der Arbeitswelt alte Sicherheiten auflöst und das subjektive Empfinden der Unsicherheit verstärkt. Der Sozialstaat hat dieser Entwicklung nicht Einhalt geboten. Im Gegenteil. Seine Neigung zur Selbstabdankung vor den risikofreudigen Unternehmen und Akteuren der Finanzwelt hat beides befördert: wachsende soziale Ungleichheit und die Krisenanfälligkeit der global vernetzten Ökonomie.

\subsection{INTERESSEN IN DER KRISE - DIE MITTELKLASSEN IM ORIENTIE- RUNGSDILEMMA}

Ein Urteil über die längerfristigen Folgen der Finanzkrise, die zugleich eine Legiti- mationskrise des Marktes ist und dessen scheinbare Naturgesetze infrage stellt, ist sicher noch verfrüht. Allerdings zeichnet sich schon heute ab, dass die Kosten der Krise unter Schonung ihrer „schöpferischen Zerstörer" auf breiter Front in die lohnabhängige Mitte und nach unten weitergereicht werden. Das wirft die Frage auf, wie zum einen die Mittelklassen reagieren, wenn die Mehrheit, die nicht als Sieger geboren, sondern zu Siegern erzogen wurde, in die Zone der Verwundbarkeit gerät. Vielleicht werden sich die bislang noch vorherrschenden sozialstaatlichen Grundüberzeugungen unter dem Druck der Finanzkrise noch fester in den lohnabhängigen Mittelklassen verankern. Aber es besteht auch die Gefahr, dass plötzlich vom Abstieg bedrohte Menschen denen da oben in der ohnmächtigen Form des Neides, der nichts bewegt, verbunden bleiben und damit nur die Herrschaft der Eliten befestigen. In den individuellen Überlebensübungen der gefährdeten Mittelklassen kann die Illusion der Selbstverantwortung in politische Apathie umschlagen, der selbst die arbeitenden und arbeitslosen Unterklassen in ihrer Resignation noch einen Rest an kollektiven Einsichten voraushaben.

Wie werden zum anderen diejenigen, die von der sich künftig verschärfenden Sozialkrise am meisten betroffen sind, ihre individuellen Situationen und gesellschaftlichen Erfahrungen verarbeiten? Welche sozialen Interessen und gesellschaftlichen Gerechtigkeitsvorstellungen könnten die Menschen in der sozialen Mitte mit jenen in den Zonen der Armut und der Prekarität verbinden: mit Dauerarbeitslosen und arbeitenden Armen, die bislang weder in den Gewerkschaften noch in den Medien eine eigene Stimme haben? Der Boulevard interessiert sich allenfalls für schaurige Bilder (über Gewalt, persönliches Elend oder ethnische Klischees), und engagierte Journalisten widmen ihnen ein paar hilfreiche Reportagen. Aber als mögliches Subjekt eines kollektiven Willens oder gemeinsamer Interessen, kurzum als soziale Klasse, kommen die in Armut und prekären Umständen Lebenden nicht vor (Ehrenreich 2009).

Das offensichtliche Fehlen einer Bewegung, die maßgebliche Teile der modernen Mittelschichten mit denen „da unten“ verbinden und das verbreitete Unbehagen an der zerstörerischen Mechanik des Finanzmarkt-Kapitalismus in konkrete Forderungen übersetzen könnte (vgl. Huffschmid 2007, 2009; Dullien et al. 2009; UN 2009), 
lässt sich nicht allein interessenlogisch damit erklären, dass der Aldi-Einkaufsmanager und nicht die Teilzeit-Kassiererin das von deutschen Banken angedrehte Lehman-Zertifikat abschreiben musste. Denn wenigstens für eine Weile saß der Schock so tief, dass die Wut über die Prellereien in der Finanzwelt das Volk der Bankgeschädigten in aufgeklärter Ohnmacht vereinte. Das ermutigt immerhin dazu, die gemeinsamen Interessen der unteren und mittleren Schichten auszuloten. Wohin könnten sie sich im Gefolge der Finanzkrise bewegen? Wenn es stimmt, dass gerade das auf die Gesellschaft bezogene Denken und Handeln der professionellen Mittelklassen von jeher individualistischer geprägt ist als das der übrigen Arbeitnehmerschichten und der arbeitslosen Armen, dann dürfte sich auch unter dem Druck einer der Finanzkrise folgenden Sozialkrise keine eindeutige Entwicklung abzeichnen. Zwei Grundorientierungen seien zur Diskussion gestellt.

\subsection{ZWEI SZENARIEN}

\section{DIE PESSIMISTISCHE VARIANTE - VERTEILUNGSKÄMPFE}

Die pessimistische Annahme geht dahin, dass unter dem Druck der Krisen und der staatlichen Finanzierungsprobleme auch in der lohnabhängigen Mittelklasse das partikulare Interesse die Oberhand gewinnt: Die Belétage ihrer Wohlhabenden würde danach auf die individuelle Absicherung des angehäuften sozialen Kapitals setzen, worauf auch jüngste Studien zum Paarungsverhalten hinweisen (Skopek et al. 2009). An diese Mentalität einer selbstgewissen Leistungselite appelliert die Klientelpolitik der FDP weit über die Kreise der Apotheker, Immobilienhändler und Anwälte hinaus. Das würde auch bei den angestellten Kadern des mittleren Managements bei verschärfter Konkurrenz um Titel und Stelle eine Mentalität befestigen, der die alte Frage nach der sozialen Gerechtigkeit abhanden kommt oder die sich auf moderne Weise sozialdarwinistisch begründet. Diese auch im akademischen Milieu der Exzellenzfanatiker vorherrschende Stimmungslage fängt Ian McEwan (2005, S. 104f.) im Profitcenter des Finanzkapitals, in London, ein. Der Mann der Mittelklasse verdrängt seine Ängste, indem er die alte Frage der Gerechtigkeit, wenngleich nicht ohne leises Bedauern, endgültig bei- seite schiebt: „Die meisten Menschen neigen zu einer fatalistischen Haltung - wer zum Lebensunterhalt die Straße fegen muss, hat einfach Pech gehabt. Wir leben in keinem visionären Zeitalter. Die Straßen müssen gesäubert werden. Sollen sich die Pechvögel darum kümmern". In diesem Sinne könnte es sein, dass die (noch) Bessergestellten die ihnen (noch) günstigen Regeln der Erfolgsspiels so lange wie möglich zu befolgen suchen.

Soziale Polarisierung und Konkurrenz würden sich aber auch innerhalb der sozial abgestuften Segmente verschärfen - für privat und öffentlich rabiatere Verkehrsformen gibt es schon ernste Anzeichen. In den anwachsenden Zonen der Verwundbarkeit erleben vor allem die jüngeren Aufsteiger eine Unsicherheit, deren Überwindung das Goldene Zeitalter des Kapitalismus allen Leistungswilligen noch versprochen hatte. Die darin enthaltene Drohung gegen die Unterklassen könnte in einem erkaltenden Sozialklima ihre Wirkung entfalten.

Eine weitere Gefahr ist, dass der moderne Typus der Klassengesellschaft mit ihren Drehtüreffekten und wechselnden Mitgliedschaften das Spektrum der Betroffenen ständig umsortiert. Damit schwinden auch in scheinbar sicheren Mittelklasseberufen die praktischen Möglichkeiten gemeinsamen Handelns, das immer ein Mindestmaß an stetigen sozialen Beziehungen benötigt. Die neuen Spielregeln des individuell nicht erarbeiteten, sondern zugeschriebenen Erfolgs sind darauf angelegt, der sozialen Einbettung des Leistungsprinzips, das die Gerechtigkeitsvorstellungen in der arbeitnehmerischen Mitte noch prägt, die Grundlage zu nehmen. Umgekehrt könnte die Finanzmarktkrise bewirken, dass die ideologische Kraft dieses inhaltsleeren Erfolgsprinzips zu schwinden beginnt.

\section{DIE OPTIMISTISCHE VARIANTE - SOZIALSTAATLICHER WERTE- KONSENS}

Eine optimistischere Sichtweise des Problems kann sich auf eine Fülle von Meinungsumfragen in Deutschland berufen, deren Ergebnisse bis kurz vor der Bundestagwahl 2009 eine große Beharrlichkeit der sozialstaatlichen Grundorientierung belegen. Selbst während des Fernsehduells Merkel/Steinmeier, das auf den direkten Kandidatenvergleich zugeschnitten war, hat eine Reaktionsanalyse an 200 Testzu- schauern die überragende Bedeutung des Themas der sozialen Gerechtigkeit bestätigt (Bohsem 2009). Dieser Befund wie auch gleichlautende Forschungsergebnisse (z. B. Sachweh et al. 2009) zeigen, dass die arbeitnehmerische Mitte ein Bedürfnis nach einem sozialstaatlichen Rahmen geltend macht. Sie könnte sogar unter dem Schock der Finanzkrise eine stärkere Rolle des Staates anmahnen, und dies nicht nur zur eigenen Statussicherung, sondern um ein Mindestmaß an sozialer Gerechtigkeit und gesellschaftlichem Zusammenhalt zu bewahren. Dieser in den Mittelklassen breit vertretene und mit den sozialen Interessen der Unterklassen vereinbare Grundgedanke einer Verbindung des Leistungs- mit dem Solidarprinzip erscheint bislang kaum geschwächt. Erwartet wird, dass auch in der entgrenzten, mit sozialen Risiken verstärkt behafteten Arbeitswelt ein faires Maß an Anerkennung der persönlichen Leistung, an „objektivierbaren“ Ungleichheiten und sozialstaatlichen Garantien besteht, das Sicherheit bei unverschuldeten Folgen von Flexibilisierungsprozessen gewährt. Soweit gibt es einen Konsens. Und selbst wenn er in Zeiten zusammenbrechender Stabilitätserfahrungen vorerst noch keine kollektive politische Aufbruchstimmung erzeugt, so kann er doch zum Impuls für Solidarität werden - zumindest dann, wenn das Empfinden der Ohnmacht gegenüber unbegriffenen Einflusskräften und Akteuren des globalen Wirtschaftens der Besinnung auf gemeinsame Interessen weicht. Immerhin herrscht schon heute unterhalb der in stabilem Wohlstand Lebenden die gemeinsame Sorge darüber vor, künftig könnten die staatlichen Transferleistungen und die privat aufgebrachten Mittel der persönlichen Vorsorge nicht mehr reichen.

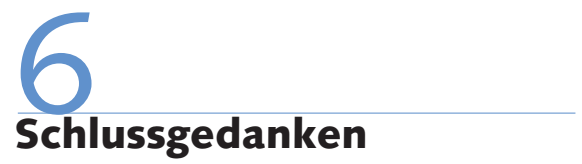

Unstrittig war der Ausbruch der Finanzkrise 2008 ein epochales Ereignis, das den „Systemirrtum“ des Finanzmarktkapitalismus so überwältigend demonstrierte, dass es für eine Weile den herrschenden ökonomischen Denkschulen die Sprache verschlug, die sie mittlerweile mit der Rede vom Staatsversagen wiederzufinden versuchen. Wenn aber die Arbeitsgesellschaft des Finanzmarkt-Kapitalismus, wie gezeigt, schon vor der Krise die Beschäftigungsfor- 
men entstandardisiert und mit der Armut massive soziale Ausgrenzungsprozesse in Gang gesetzt hat, dann wäre die Zeit nach der Krise die Versuchsphase für eine Re-Regulierung nach politisch zu bestimmenden sozialen Interessen. Dass diese Schockphase, wie nicht nur das Wahlergebnis in Deutschland zeigt, nicht genutzt wurde, dass ferner die gesellschaftlichen Folgeprobleme sich - nach einer Zeit des Hinausschiebens - verschärfen werden, ist nüchtern zu konstatieren. Das Nachdenken über die Gründe kann aber auch Kräfte für ein anderes Handeln freisetzen.

Wie ließe sich der derzeitige gesellschaftliche Zustand des gleichgültigen Nebeneinanders von Unter- und Mittelschicht überwinden, der sich u.a. bei der Bundestagswahl im September 2009 in der hohen Quote der Nichtwähler aus den gesellschaftlichen Armutszonen ausdrückte? Dafür schlägt Kronauer einen Weg vor, der sowohl Unter- als auch Mittelklassen eine gemeinsame Perspektive weisen könnte. Seine Kritik an einer nur formalen Integration der Menschen, die in bestehenden Ungleichheitsstrukturen leben, gilt den konkreten Institutionen, die solche Strukturen befestigen und die Individuen ohnmächtig halten. Wenn „am Arbeitsmarkt, in den Arbeitsverhältnissen, in den Systemen der sozialen Sicherung, der Vermittlung von Bildung und Gesundheit Möglichkeiten der Ausgrenzung ... institutionalisiert sind, überfordert dies das individuelle Gegensteuern“ (Kronauer 2010, S. 56).
Gefragt ist als Antwort eine Politik der Inklusion, welche „die ausgrenzenden Institutionen selbst infrage stellt" (ebd.). Eine neu zu erfindende Politik könnte z. B. die Neuverteilung der Arbeitszeit fördern, mit dem Ziel, eine gerechtere Verteilung anzustreben, anstatt 60 Wochenstunden für einige und null für andere. Oder sie könnte mit einer gesellschaftlichen Verteilung der Lebenschancen brechen, die eine wachsende Zahl von Menschen ausschließt, demütigt und ihre Lebensgeschichte entwertet.

Welche Argumente sprechen für mehr Gleichheit in der Gesellschaft? In den USA hat Robert H. Frank (2007) den Kern des Problems im Untertitel seiner Studie festgehalten: „How Rising Inequality Harms the Middle Class". Deren zum ständigen Vergleich erzogene Mitglieder reagieren auf den wachsenden Abstand zu den Oberklassen mit einem neurotisierten Leistungsund Konsumverhalten, das sie im Durchschnitt nur noch missgünstiger macht. Aber warum sollte, um dies Unglück abzuwenden, ein maßgeblicher Teil der Mittelschichten, der mit dem Risiko sozialer Verwundbarkeit konfrontiert ist, mit den Unterklassen gemeinsame Sache machen und sich um etwas kümmern, das sie scheinbar nichts angeht? Welches Interesse sollte der junge Betriebswirt, der nach dem zweiten Praktikum seine erste bezahlte Stelle mit Glück ergattert hat, daran haben, von seinem gehobenen Einkommen vernünftig Steuern zu zahlen oder gar im Unterneh- men gegen Leiharbeit und für den Vertragsabschluss mit einer Fremdfirma zu plädieren, die dem Reinigungspersonal den Mindestlohn zahlt?

Die Frage berührt das in der Gerechtigkeitsforschung erörterte Verhältnis des Anspruchs auf individuelle Selbstgestaltung und soziale Sicherheit. Der ökonomische Liberalismus in seinem Misstrauen gegenüber dem Menschen als kooperativem Wesen opfert das schwer zu verwirklichende Leitbild sozialer Gerechtigkeit dem Primat des freien Individuums, das sich auch in der Freiheit der Ausbeutung Dritter verwirklichen darf und vor allem Verteilungsgleichheit als effizienzfeindlich strikt ablehnt. Diese Unvereinbarkeitserklärung ist Ideologie und kann sich, wie Miller (2008, S. 104ff.) überzeugend darlegt, keineswegs auf die in der Bevölkerungsmehrheit verbreiteten Gerechtigkeitsvorstellungen berufen, die mit dem Leistungsprinzip der Mittelklassen vereinbar sind. Auch Wilkinson/Pickett (2009) ermutigen mit ihren empirischen Forschungen zu einem Gesellschaftsprojekt, das auf die Vereinbarkeit der Interessen von Mittel- und Unterschicht setzt. Sie zeigen an minutiös erhobenen Daten, dass stärker egalitäre Gesellschaften schlicht besser funktionieren und die Mehrheit ihrer Mitglieder glücklicher machen als die angsterfüllten Reichen in ihren gated communities es sind. Das ist nicht mehr, aber auch nicht weniger als eine schöne Vorstellung, die ein kleines Tor zum alltäglichen Handeln öffnet.

\section{LITERATUR}

Bohsem, G. (2009): Publikum votiert für Gerechtigkeit, in: Süddeutsche Zeitung vom 15. September, S. 5

Brenke, K. (2009): Reallöhne in Deutschland über mehrere Jahre rückläufig, in: DIW-Wochenbericht 33, S. 550-560

Bude, H. (2008): Die Ausgeschlossenen. Das Ende vom Traum der gerechten Gesellschaft, München

Deutschmann, C. (2005): Finanzmarkt-Kapitalismus und Wachstumskrise, in: Windolf, P. (Hrsg.): Finanzmarkt-Kapitalismus. Analysen zum Wandel von Produktionsregimen, Wiesbaden, S. 58-84

Dullien, S./Herr, H./Kellermann, C. (2009): Der gute Kapitalismus... und was sich dafür nach der Krise verändern müsste, Bielefeld

Ehrenreich, B. (2009): Too Poor to Make the News, in: The New York Times, June 14

Frank, R. H. (2007): Falling Behind: How Rising Inequality Harms the Middle Class, Berkeley

Frick, J. R./Grabka, M. M. (2009): Gestiegene Vermögensungleichheit in Deutschland, in: DIW-Wochenbericht 4, S. 54-67
Ganßmann, H. (2006): Kapital - Arbeit, in: Lessenich, S./Nullmeier, F (Hrsg.): Deutschland - eine gespaltene Gesellschaft, Frankfurt a. M./New York, S. 92-114

Grabka, M. M./Frick, J. R. (2008): Schrumpfende Mittelschicht - Anzeichen einer dauerhaften Polarisierung der verfügbaren Einkommen?, in: DIW-Wochenbericht 10, S. 101-108

Groh-Samberg, O. (2007): Armut in Deutschland verfestigt sich, in: DIWWochenbericht 12, S. 177-182

Groh-Samberg, O. (2009): Armut, soziale Ausgrenzung und Klassenstruktur. Zur Integration multidimensionaler und längsschnittlicher Perspektiven, Wiesbaden

Huffschmid, J. (2007): Jenseits der Spekulationskrise. Finanzgetriebener Kapitalismus und Perspektiven der Gegensteuerung, in: Blätter für deutsche und internationale Politik 11, S. 1331-1341

Huffschmid, J. (2009): The Crash reloaded?, in: Blätter für deutsche und internationale Politik 2, S. 110-112 
International Labour Organisation (ILO) (2009): Global Employment Trends - Update May, Geneva

Kädtler, J. (2005): Finanzmärkte - zur Soziologie einer organisierten Öffentlichkeit, in: SOFI-Mitteilungen 33, S. 31-37

Kronauer, M. (2010): Inklusion - Exklusion. Eine historische und begriffliche Annäherung an die soziale Frage der Gegenwart, in: Ders. (Hrsg.): Inklusion und Weiterbildung. Reflexionen zur gesellschaftlichen Teilhabe in der Gegenwart, Bielefeld, S. 24-58 (im Erscheinen)

Krugman, P. (2002): For Richer, in: The New York Times Magazine, 10/20 Lessenich, S./Nullmeier, F. (Hrsg.) (2006): Deutschland - eine gespaltene Gesellschaft, Frankfurt a. M./New York

McEwan, I. (2005): Saturday, Zürich

Miller, D. (2008): Grundsätze sozialer Gerechtigkeit, Frankfurt

a. M./New York

Misik, R. (2009): Krise? Welche Krise?, in: Die Tageszeitung vom 11.

September 2009

Paoli, G. (2001): Lasst euch nicht gehen. Weisheiten der Kampfkunst, in: Hegemann, C. (Hrsg.): Erniedrigung genießen. Kapitalismus und Depression III, Berlin, S. 60-84

Philippon, T./Reshef, A. (2009): Wages and Human Capital in the U. S. Financial Industry. Working Paper 14644, National Bureau of Economic Research
Sachweh, P./Burkhardt, C./Mau, S. (2009): Wandel und Reform des deutschen Sozialstaats aus Sicht der Bevölkerung, in: WSI-Mitteilungen 11, S. 612-618

Skopek, J./Schulz, F./Blossfeld, H.-P. (2009): Partnersuche im Internet. Bildungsspezifische Mechanismen bei der Wahl von Kontaktpartnern, in: Kölner Zeitschrift für Soziologie und Sozialpsychologie 2, S. 159-182 Thielemann, U. (2009): Mit Unternehmensethik gegen den System Error des "freien" Marktes? Vortrag an der Hochschule für Wirtschaft und Recht (HWR) Berlin, am 29. Oktober

United Nations (UN) (2009): Report of the Commission of Experts of the President of the United Nations General Assembly on Reforms of the International Monetary and Financial System, September 21

Vogel, B. (2009): Wohlstandskonflikte. Soziale Fragen, die aus der Mitte kommen, Hamburg

Wilkinson, R./Pickett, K. (2009): The Spirit Level. Why More Equal Societies Almost Always Do Better, Harlow

Windolf, P. (2005): Was ist Finanzmarkt-Kapitalismus?, in: Ders. (Hrsg.): Finanzmarkt-Kapitalismus. Analysen zum Wandel von Produktionsregimen, Wiesbaden, S. 20-57 